\title{
Áreas naturales protegidas e investigación científica en el Perú
}

\author{
Protected areas and scientific research in Peru
}

\author{
Marc J. Dourojeanni ${ }^{1, *}$
}

\begin{abstract}
Resumen
Desde sus orígenes, en los años 1960, el sistema de áreas naturales protegidas del Perú ha sido diseñado en función de criterios científicos. La teoría de las zonas de vida natural de L. Holdridge orientó la selección de las primeras áreas protegidas, complementada por informaciones sobre especies endémicas y amenazadas. Posteriormente pasó a usarse la técnica de las evaluaciones ecológicas rápidas. Las áreas protegidas han sido intensamente usadas por científicos como lo demuestra el gran número de investigaciones ecológicas y la alta proporción de especies nuevas descubiertas en ellas, destacando el trabajo realizado en la Estación Ecológica de Cocha Cashu en el Parque Nacional Manu. Pero los resultados han sido poco aprovechados para el manejo de las áreas. Aunque hay conflictos entre la gestión de las áreas protegidas y las necesidades de los científicos, en general existe una relación armoniosa y mutuamente benéfica.
\end{abstract}

Palabras clave: Áreas naturales protegidas, ciencia, investigación científica.

\footnotetext{
${ }^{1}$ Facultad de Ciencias Forestales, Universidad Nacional Agraria La Molina (UNALM), Av. La Molina s/n, La Molina, Lima, Perú.

*Autor de Correspondencia: marc.dourojeanni@gmail.com
} 


\begin{abstract}
Since its origins, in the 1960s, the system of natural protected areas of Peru has been designed according to scientific criteria. The theory of natural life zones of L. Holdridge oriented the selection of areas to be included in the early protected areas system, supplemented by information on endemic and threatened species. Subsequently the technique of rapid ecological evaluations has usually been applied. The protected areas have been intensely used for ecological research and for taxonomic research as shown by the high proportion of new species discovered. The Cocha Cashu Ecological Station in the Manu National Park has been very prolific in high level continued research work. However, research results are scarcely used for management. Although there are conflicts between the management of protected areas and scientists' needs, in general there is a harmonious and mutually beneficial relationship.
\end{abstract}

Key words: Protected areas, science, scientific research.

\section{Introducción}

Las relaciones de las áreas protegidas con la ciencia se pueden examinar tanto desde el ángulo de la ciencia como desde el de las áreas protegidas. La ciencia contribuye a las áreas protegidas en dos formas: brindando justificaciones para su establecimiento y proporcionando informaciones para su manejo efectivo. Las áreas protegidas ofrecen a la ciencia lugares apropiados para su realización, especialmente por ser muestras naturales y por tener menos riesgos de disturbio que otros lugares. Además son espacios más seguros para los investigadores. Pero hay otros temas importantes que relacionan áreas protegidas y ciencia, como el límite que la conservación impone a la ciencia y que puede incomodar a los científicos $\mathrm{y}$, asimismo, no puede dejar de mencionarse que hay conocimientos y descubrimientos científicos que pueden crear problemas a las áreas protegidas. En esta nota se revisan esas relaciones en el contexto de las áreas naturales protegidas del Perú.

\section{Establecimiento de áreas protegidas ¿la ciencia como justificación o como pretex- to?}

Prácticamente todas las áreas naturales protegidas del Perú creadas después de 1965 se establecieron a partir de argumentos esencialmente científicos. En el Perú el argumento principal, usado desde que se diseñó el primer plan del sistema (Pierret 1964, 1965; Grimwood 1967; Dourojeanni 1968; Dourojeanni y Ponce 1977), fue asegurar una representatividad ecológica adecuada, basada en la teoría de la ecología de las zonas de vida natural desarrollada por Holdridge (1947) y aplicada en el Perú por Tosi (1960), que en principio debería cubrir lo esencial de la diversidad biológica nacional. Complementariamente se introducían consideraciones sobre especies raras, amenazadas o en peligro de extinción (Grimwood 1968). No obstante, esos mismos autores también llevaron en cuenta justificaciones complementarias, como los paisajes naturales nacionalmente famosos (Huascarán) o el patrimonio arqueológico (Machu Picchu), entre otros. Hubo casos, como Lachay y Pacaya-Samiria, que ya estaban en cierta forma escogidos desde varias décadas antes aunque también basado en cuestiones científicas (Aguilar 1963; Piazza 1967). En realidad, los dos primeros parques nacionales del Perú (Cutervo y Tingo María) aunque pobremente diseñados, usaron asimismo argumentos científicos para justificarse, en esos casos la protección de una ave amenazada, Steatornis caripensis (Vilchez 1968; Dourojeanni y Tovar 1972).

Ya en esos días se sugería que el establecimiento de las áreas naturales protegidas de la Selva podría haberse fundamentado en otros criterios, como el de la teoría de los refugios del 
Pleistoceno (Haffer 1969; Lamas 1979), que los brasileños usaron para planear sus áreas protegidas amazónicas (Pádua y Coimbra-Filho 1979). Pero la geografía accidentada del Perú, inclusive de su región amazónica, mostraron que no era necesario aplicar ese criterio ya que el sistema Holdridge lo cubría muy bien. Los argumentos científicos pasaron a tener aún más importancia para proponer nuevas áreas protegidas a lo largo de la década de 1980 . Por ejemplo, fueron usados para las propuestas de establecimiento del Parque Nacional Abiseo creado en 1983 (Mittermeier et al. 1977; Leo 1982), del Santuario Nacional del Heath creado en 1983 (Hofman et al. 1976); de Junín, creado en 1975 (Dourojeanni et al. 1968), entre muchos otros.

A partir de la década de 1990 se usaron metodologías justificativas más detalladas. Apareció la metodología de las evaluaciones ecológicas rápidas (Sayre et al. 1999) que se usó para prácticamente todas las áreas protegidas creadas en el Perú desde entonces, como los parques nacionales Bahuaja-Sonene (Foster et al. 1994, Montambault, 2002), Ichigkat-Muja (Schulenberg y Awbrey 1997), Otishi (Alonso et al. 2001), Sierra del Divisor (Vriesendorp et al. 2006) o Yaguas, el último establecido (Pitman et al. 2004). Pero las justificaciones se basaron asimismo en extensas referencias científicas acumuladas a lo largo de las décadas. Ver, por ejemplo, Tovar et al. (2009) para Sierra del Divisor. Hay otros casos en que la ciencia alertó sobre situaciones específicas que necesitan atención. En el Perú ese ha sido el caso de los ecosistemas de arenas blancas (Fine et al. 2010) que dieron lugar a la Reserva Nacional Allpahuayo-Mishana.

Otra forma en que desde mediados de los años 1980 las áreas naturales protegidas han aprovechado de la ciencia para su establecimiento y, asimismo, manejo, fue la creación del Centro de Datos para la Conservación (CDC) en la Universidad Nacional Agraria la Molina ${ }^{1}$ que acumula, procesa, actualiza y distribuye información valiosísima sobre la diversidad bio-

\footnotetext{
${ }^{1}$ http://cdc.lamolina.edu.pe/QuienesSomos/historia.html
}

lógica nacional, su distribución y situación. El CDC ha contribuido directa o indirectamente a brindar fundamentos científicos para la creación de nuevas áreas protegidas en el país.

Es decir que en el caso peruano no parecen totalmente aplicables las conclusiones del estudio de Baldi et al. (2017) que afirma que lo que ha orientado la distribución mundial de las áreas protegidas es la oportunidad y no la representatividad ambiental ni la protección de la biodiversidad. Ese análisis argumenta que el aislamiento y la baja densidad de población serían los factores preponderantes de selección. Puede ser, ya que no es posible crear áreas protegidas donde el uso de la tierra ya determinado es incompatible con la conservación. Sin embargo, en el Perú el aislamiento y la población reducida han sido elementos suplementarios o complementarios al científico. Sin embargo, hay países que en efecto han escogido gran parte de sus áreas protegidas usando criterios tales como el geopolítico y el estético, como lo hizo el Perito Moreno en Argentina. Otro estudio (Fajardo et al. 2014) afirma haber identificado grandes deficiencias en la representatividad ecológica del sistema peruano de áreas protegidas. Esas existen, sin duda, aunque quizá no sean tan significativas como lo pretenden esos autores y es preciso esforzarse para disminuirlas. Para eso se cuenta, por ejemplo, con el concepto de corredores biológicos o ecológicos, complementado por el establecimiento de reservas privadas u concesiones de conservación y turismo, lo que debe ser aplicado con más énfasis.

Los científicos de la actualidad frecuentemente olvidan que establecer áreas protegidas es también el arte de lo posible. Al final, se trata de complejas decisiones políticas. Tampoco llevan en cuenta, en sus críticas, que cuando se estableció la base del sistema de áreas protegidas no existían las herramientas que ahora son de uso común como información satelital, computadoras portátiles, GPS y telefonía celular. Además, no se puede dejar de lado el hecho de que la decisión política de crear áreas protegidas es tomada por personas que no siempre entienden el significado de las argu- 
mentaciones científicas. Por eso, tanto pueden aceptar justificaciones que parecen científicas como pueden alterar arbitrariamente las propuestas, especialmente los límites propuestos, lo que es muy frecuente (Dourojeanni 2018).

En conclusión, por lo menos en el Perú, no hay evidencia de que la ciencia fue usada como pretexto para establecer áreas naturales protegidas. Fue su justificación.

\section{Simbiosis entre ciencia y áreas protegidas}

La ciencia brinda mucho más a las áreas protegidas que apenas elementos justificativos para su establecimiento. Aporta, en principio, informaciones indispensables para su manejo. Estas incluyen desde cuestiones como tamaño mínimo, crítico o viable (Lovejoy y Oren 1981; Laurance et al. 2011) e inventarios biológicos de lo que contiene cada área, hasta análisis de opciones para enfrentar el cambio climático, y medir los riesgos de extinción de medio y largo plazo, entre tantos otros asuntos. También provee información para evaluar la calidad del manejo, las relaciones entre el área protegida y la sociedad del entorno, la densidad de visitación tolerable para cada ecosistema o hábitat, etc.

En áreas en las que se conserva y maneja poblaciones animales o vegetales la dependencia es aún mayor, como es bien conocido desde hace décadas en el caso del manejo de la vicuña en Pampa Galeras (Franklin 1976; Hofmann et al. 1983) y del venado cola blanca y otras especies en el Coto de Caza El Angolo (Vásquez y Justo 2009; Elías y Vásquez 2016; Olivera y Vásquez 2017). Más antiguo aún ha sido el aporte de grandes científicos como W. Vogt, E. Schweiger y H.O. Forbes para el manejo de las aves guaneras en lo que hoy es el Sistema de Islas, Islotes y Puntas Guaneras. A priori no es posible manejar adecuadamente un área protegida sin disponer de información científica. Un ejemplo más reciente son los trabajos de Soini (1997) para el manejo de las tortugas acuáticas en la Reserva Nacional Pacaya-Samiria.

No obstante, se debe reconocer que debido a las restricciones presupuestales del Siste- ma Nacional de Áreas Naturales Protegidas (SINANPE) y de su ente rector, el Servicio Nacional de Áreas Naturales Protegidas (SERNANP), se aprovecha poco de lo que la ciencia ofrece a las áreas protegidas en general y a aquellas en que se realiza la investigación. Más aún cuando en ellas se establecen centros de investigación que producen grandes volúmenes de información. Tal es el caso de Cocha Cashu, en el Parque Nacional del Manu, instalado en 1967 a iniciativa de la Universidad Nacional Agraria de la Molina (UNALM), sitio en el que se realizaron investigaciones sobre el lagarto negro (Otte 1978) desde antes de la creación del Parque. Luego, aporte de centenas de investigadores durante casi 50 años ininterrumpidos ha generado mucho más datos (Groenendijk 2013) que lo aprovechado y aprovechable para el manejo efectivo del Parque. Esto no es un problema exclusivo al Perú (Vásquez y Matallana 2016).

Lo que tampoco es discutible es que las áreas protegidas aportan mucho a la ciencia, brindando lugares donde la investigación disfruta de ecosistemas poco o nada alterados, exhibiendo toda la diversidad biológica que corresponde al hábitat así como las relaciones inter e intraespecíficas naturales. La oportunidad que esos lugares ofrecen para el descubrimiento de especies nuevas, por ejemplo, es invalorable y por eso son tan procurados. Para los que estudian la biología y la ecología sobre plazos largos las áreas protegidas ofrecen también la ventaja de que los locales de estudio se mantienen sin riesgo de intromisiones o alteraciones (Maass et al. 2010) y, asimismo, aportan mayor seguridad para la integridad física de los propios científicos.

Por eso las áreas protegidas son tan procuradas para la realización de investigaciones, en especial taxonómicas. Apenas en 2017 fueron descritas cuatro especies nuevas en el Abiseo (Rodriguez y Catenazzi 2017), tres en Cordillera Azul (Lane et al. 2017; Cusi et al. 2017; Moncrieff et al. 2017), una en Pui Pui (Lehr y von May 2017); una en Tingo María (Chávez y Catenazzi 2016), una en Tabaconas-Namballe (Zeballos et al. 2018), y eso es apenas una 
muestra. También se registraron tres especies nuevas para el Perú en Bahuaja-Sonene (Medina et al. 2016). Si se hiciera un inventario de todas las especies de animales y plantas encontradas en áreas protegidas desde los años 1960, que son nuevas para la ciencia o que son nuevos registros para el Perú, se demostraría que son mayoría. Gran parte del descubrimiento, a nivel de toda la Amazonia, de 1200 especies nuevas entre 1999 y 2009 (WWF 2009) y de 441 entre 2010 y 2013 (Tickell 2013), fue logrado en áreas protegidas.

Otra forma de uso de las áreas naturales protegidas, especialmente las de uso indirecto, ha sido como áreas testigo de los cambios provocados por la actividad humana y el desarrollo económico. Ese fue el origen de las llamadas reservas de biosfera y del Programa del Hombre y la Biosfera de la Unesco (Batisse 1980, 1986), de las que el núcleo es generalmente constituido por parques nacionales. En el Perú hay varias reservas de biosfera pero, otra vez por falta de fondos esa función central de las reservas de biosfera nunca funcionó y hoy se ha perdido.

La ciencia es esencial para mejorar o refinar la representatividad ecológica del SINANPE tal como es ahora (Fajardo et al. 2014) y, en especial, para el diseño de los corredores biológicos o ecológicos llenando los espacios entre áreas protegidas existentes, manteniendo o creando la conectividad necesaria para mantener los flujos genéticos (Bennet 1999). Estos corredores son asimismo una de las pocas herramientas disponibles para mitigar el impacto del cambio climático en las laderas andinas, permitiendo que subsista una migración adaptativa (Krosby et al. 2010).

\section{¿Ciencia contra las áreas naturales prote- gidas?}

La comunidad científica a menudo ha lanzado puntos de vista que proporcionaron argumentos a los responsables políticos que se oponen al establecimiento de áreas protegidas. Un enfrentamiento generalizado entre las opiniones de los científicos naturales y sociales sobre la naturaleza y la conservación de la naturaleza viene comprometiendo los logros de la conservación. Desde finales de la década de 1970, pero especialmente después de la consagración del concepto de desarrollo sostenible (WCED 1987), la crítica de los científicos sociales contra las áreas estrictamente protegidas aumentó inclusive ridiculizando las áreas protegidas con encuestas tendenciosas como la de Machlis y Tichnell (1985) que permitieron acuñar el slogan "parques de papel", a menudo simplemente invirtiendo el sentido de un mismo argumento científico, como en el caso del aislamiento (Diegues 1996; Quammen 1996). Esta tendencia ha estado en constante crecimiento (Gómez-Pompa y Kahm 1992), evolucionando hacia el antropocentrismo acentuado de hoy en día que, en muchos sentidos, se une a posiciones que también son defendidas por los desarrollistas (Kareiva et al. 2012). También han aparecido otras voces, supuestamente basadas en datos científicos, que recomiendan, literalmente, "dejar que algunos animales se extingan" aduciendo el alto costo que tiene evitarlo (Ferry 2017). Esto ha sido fundamental para el establecimiento de una serie de nuevas áreas "protegidas" abiertas a la ocupación humana y a la utilización de sus recursos (Allegretti 2008). Por un lado más positivo, los científicos sociales obviamente han influenciado en el desarrollo de la llamada "conservación basada en la comunidad" y, como se esperaba, fueron determinantes en la mayor prioridad otorgada al reconocimiento de los derechos de los pueblos indígenas.

Antes de la década de 1970 el concepto predominante sobre la capacidad de uso de la tierra de la Amazonia era que la región tenía un potencial muy limitado para la agricultura de labranza en limpio y bastante limitada para cultivos y pastos permanentes (National Research Council 1982). La mayoría de los estudios estimaban que la capacidad de la Amazonia para la agricultura y la ganadería era inferior al 11\% (Zamora 1971). Esto se ha reflejado en la legislación de países como Perú, que restringe la deforestación y facilitaba el establecimiento de áreas naturales protegidas. 
Sin embargo, la mayoría de los científicos de suelos y agrónomos criticaron este punto de vista y respaldaron el principio de que las limitaciones naturales de los suelos amazónicos se pueden superar fácilmente con tecnología apropiada que depende esencialmente de la economía (Sanchez y Buol 1975). A pesar de las dudas justificadas sobre su sostenibilidad (Fearnside 1987), esta tendencia ha dominado, y grandes porciones de la Amazonia, especialmente en Brasil, ahora se utilizan para la agricultura mecanizada intensiva. Además, los avances en las ciencias y tecnologías agrícolas, incluido el control de malezas y plagas, la gestión de suelos y las plantas genéticamente mejoradas, entre otros avances, aunados a la creciente demanda mundial de alimentos y a la nueva infraestructura de transporte, hicieron de la agricultura tropical intensiva una realidad económicamente viable. Es decir que nuevas áreas protegidas, inclusive en esa región, ahora también confrontan los intereses de la gran agricultura de exportación.

No menos importantes fueron los enormes progresos realizados en materia de teledetección, que incluyen el uso de drones, comunicaciones, tecnologías de construcción y muchos otros que, por supuesto, pueden ser útiles para conservar la Amazonia, pero que son igualmente un activo para profundizar su explotación, como en el caso de los estudios geológicos que revelaron la riqueza de minerales y de hidrocarburos. Como bien se sabe, las concesiones petroleras han sido y son el principal obstáculo para el establecimiento de áreas protegidas en la Selva (Sierra del Divisor, Yaguas) y en el litoral Norte, como lo fueron los minerales en el caso del Parque Nacional Ichigkat-Muja.

\section{Conflictos entre ciencia y áreas protegi- das}

La ley $\mathrm{N}^{\circ} 26834$ del 2000 reconoce la importancia de que las áreas naturales protegidas estén disponibles para la investigación científica y aplicada. Pero determina que ésta no debe afectar los objetivos primarios de conservación y que se adecue al plan maestro respectivo. El reglamento de la ley dedicaba un subcapítulo a los convenios y proyectos de investigación pero fue derogado en 2015, posiblemente por la complejidad de los trámites que implicaba. De hecho fue sustituido por el Decreto Supremo $N^{\circ}$ 010-2015-MINAM, que consideró la investigación en áreas protegidas como de interés nacional, actualizó el proceso de solicitud de autorizaciones de investigación y dispuso que el trámite sea gratuito, más simple y breve. Aparentemente aún existen muchas quejas por parte de los investigadores, especialmente referentes al trámite y al poco apoyo que reciben durante el trabajo de campo. El caso no es peor en el Perú que en los demás países.

Ocurre que la llegada de investigadores a los parques suele complicar aún más la administración del área, ya bajo presión por los conflictos habituales, debiendo compartir la pobreza de la infraestructura y de los medios logísticos con los recién llegados. A eso se suma el carácter frecuentemente crítico de los investigadores que puede crear situaciones tensas con el personal del área protegida. De otra parte, muchas de las investigaciones que se realizan en áreas protegidas no ofrecen ningún beneficio evidente o directo para mejorar el manejo, y por lo que el personal del área no se siente compelido a apoyarlas.

Este autor ha testimoniado investigadores que abusan de sus privilegios ocasionando riesgos a determinadas poblaciones debido, por ejemplo, a la recolección de números excesivos de especímenes u ocasionando elevada mortalidad entre ellos. Existe, sin duda, un riesgo mayor con investigadores novatos, muchas veces estudiantes de posgrado que no saben bien lo que hacen. El personal del área protegida no tiene tiempo ni medios para hacer seguimiento cercano de los investigadores.

Por otra parte, la presencia de científicos en las áreas naturales protegidas puede contribuir a su protección y, obviamente, a la difusión mundial de sus atributos. Kai Otte en el Manu, Pekka Soini y otros en Pacaya-Samiria, o William Franklin en Pampa Galeras, preparando sus tesis doctorales, fueron profesionales permanentemente presentes en esas áreas 
protegidas en los difíciles momentos iniciales. La presencia de John Terborgh y de sus discípulos en el Manu contribuyó a construir la fama mundial de ese Parque, así como a obtener fondos y apoyo internacional. Laurance (2013) abunda en ejemplos en que la presencia de científicos contribuye a la protección de las áreas naturales.

Otro tema asociado y que complica la vida de los investigadores científicos, dentro y fuera de las áreas naturales protegidas, es el de las normas que protegen la fauna y flora nativa de actos de biopiratería (Dourojeanni 2004) y su vinculación a la Convención sobre Comercio Internacional de Especies Amenazadas.

\section{Conclusión}

En el Perú, quizá más que en otros países, las áreas naturales protegidas se han establecido siempre en función de información científica suficientemente sólida como para justificar su necesidad ante la opinión pública y los tomadores de decisión. Esto se explica porque el Perú comenzó a crear sus áreas naturales protegidas con mucho retardo sobre los demás países de la región y del mundo, teniendo la oportunidad de evitar errores cometidos por otros. Asimismo, esas áreas han cumplido bien el rol que se les asigna para apoyar el desarrollo de la ciencia, como lo demuestra el hecho de que gran parte de la producción científica biológica y ecológica tanto nacional como extranjera sobre el Perú se ha realizado dentro de áreas protegidas. La principal deficiencia en la relación entre la ciencia y las áreas protegidas es el poco uso que se hace de los resultados de las investigaciones en la gestión, esencialmente a consecuencia de las limitaciones presupuestales para el manejo efectivo. De otra parte, aunque la ciencia se ha desarrollado en buena armonía con el manejo de las áreas protegidas es evidente que aún existe mucho por hacer para facilitar e incentivar la investigación.

\section{Bibliografía}

Aguilar, P. 1963. Los artrópodos de las lomas de los alrededores de Lima. Revista peruana de entomología agrícola 6(1):109-114.
Allegretti, M. 2008. The social construction of public policies. Chico Mendes and the seringueiros movement. Desenvolvimento e Meio Ambiente 18: 39-59.

Alonso, LE; Alonso, A; Schunlenberg, TS; Dallmeier, F. 2001. Perú: Cordillera de Vilcabamba. Biological and Social Assessments of the Cordillera de Vilcabamba, Peru. Washington, DC, USA. RAP Working Papers 12, SI/MAB Series 6. Conservation International. 296 p.

Baldi, G; Texeira, M; Martin, O; Grau, HR; Jobbágy, E. 2017. Opportunities drive the global distribution of protected areas. PeerJ 1-24. DOI: http://doi.org/10.7717/peerj.2989

Batisse, M. 1980. The relevance of MAB. Environmental Conservation 7(3):179-84.

Batisse, M. 1986 Developing and focussing the Biosphere Reserve concept. Nature and Resources XXII(3):2-11.

Bennett, AF. 1999. Linkages in the landscape: The role of corridors and connectivity in wildlife conservation. IUCN, Gland, Switzerland. $254 \mathrm{p}$.

Cusi, JC; Moravec, J; Lehr, E; Gvoždík, V. 2107. A new species of semiarboreal toad of the Rhinella festae group (Anura, Bufonidae) from the Cordillera Azul National Park, Peru. Zookeys 673:21-47. DOI: https://doi.org/10.3897/zookeys.673.13050

Chávez, G; Catenazzi, A. 2016. A new species of frog of the genus Pristimantis from Tingo María National Park, Huánuco Department, central Peru (Anura, Craugastoridae). ZooKeys 610:113-130.

Diegues, AC. 1996. O Mito Moderno da Natureza Intocada. São Paulo, Brasil. HUCITEC. $169 \mathrm{p}$.

Dourojeanni, MJ. 1968. Estado actual de la conservación de la flora y de la fauna en el Perú. Ciencia Interamericana 9(1-6):1-12.

Dourojeanni, MJ. 2004. De piratas e biopiratas (em línea). O Eco. Consultado 23 nov. 2018. Disponible en http://www.oeco.org.br/colunas/marc-dourojeanni/16335-oeco-10820/ 
Dourojeanni, MJ. 2018. Áreas Naturales Protegidas del Perú: El Comienzo. Lima, Perú. Univ. Nac. Guzmán y Valle, Editorial Grijley. 330 p.

Dourojeanni, MJ; Hofmann, R; García, R; Malleux, J; Tovar, A. 1968. Observaciones preliminares para el manejo de las aves acuáticas del Lago de Junín, Perú. Revista Forestal del Perú 2(2):3-52.

Dourojeanni, MJ; Tovar, A. 1972. Notas sobre el ecosistema y la conservación de la Cueva de las Lechuzas (Parque Nacional de Tingo María, Perú). Revista Forestal del Perú 5(1-2):28-45

Dourojeanni, MJ; Ponce, C. 1978. Los Parques Nacionales del Perú. Madrid, España. INCAFO. 224 p.

Elías, R; Vásquez, P. 2016. Protocolo Sanitario para Venados Cola Blanca (Odocoileus virginianus peruvianus) del Sector Sauce Grande Coto de Caza El Angolo, Piura, Perú. Rev. Inv. Vet. Perú 27(3): 531-538. DOI: http://dx.doi. org/10.15381/rivep.v27i3.12001

Foster, RB; Parker III, TA; Gentry, AH; Emmons, L; Chicchón, A; Schulenberg, T; Rodríguez, L; Lamas, G; Ortega, H; Icochea, J; Wust, W; Romo, M; Castillo, JA; Phillips, O; Reynel, C; Kratter, A; Donahue, PK; Barkley, LJ. 1994. Tambopata-Candamo Reserved Zone. The Tambopata-Candamo Reserved Zone of southeastern Perú: A Biological Assessment. Washington, DC, USA. RAP Working Papers 6. Conservation International. $184 \mathrm{p}$.

Fajardo, J; Lessmann, J; Bonaccorso, E; Devenish, C; Muñoz, J. 2014. Combined use of systematic conservation planning, species distribution modelling, and connectivity analysis reveals severe conservation gaps in a megadiverse country (Peru). PLoS ONE 9(12):e114367. DOI: https://doi.org/10.1371/ journal.pone.0114367

Fearnside, PM. 1987. Rethinking continuous cultivation in Amazonia. Bioscience 37(3):209213.

Ferry, D. 2107. It's time to let certain animals go extinct (en línea). Outside. Consultado 23 nov. 2018. Disponible en https://www.outside- online.com/2176276/its-time-choose-whichanimals-we-let-go-extinct

Fine, P; García-Villacorta, R; Pitman, N; Mesones, I; Kembel, S. 2010. A floristic study of the white-sand forests of Peru. Annals of the Missouri Botanical Garden 97(3):283-305.

Franklin, WL. 1976. Socioecology of the vicuna. Tesis Ph.D. Utah, USA, Utah State University. $172 \mathrm{p}$.

Gómez-Pompa, A; Kahm, A. 1992. Taming the wilderness myth. BioSciences 42(4):271-279.

Grimwood, IR. 1967. Recommendations on the conservation of wildlife and the establishment of national parks and reserves in Peru. British Ministry of Overseas development (a collection of reports).

Grimwood, IR. 1968. Notes on the distribution and status of some Peruvian mammals. Special Publication N²1, NY Zoological Society. 86 p.

Groenendijk, J (ed.). 2013. Reporte Manu 2013 - Pasión por la Investigación en la Amazonia Peruana. Lima, Perú. San Diego Zoo Global Peru y SERNANP. 466 p.

Haffer, J. 1969. Speciation in Amazonian forest birds. Science 165:131-137.

Hofmann, R; Ponce, CF; Otte, KC. 1976. Registro de dos nuevas especies de mamíferos para el Perú, Odocoileus dichotomus (Illiger, 1811) y Chrysocyon brachyurus (Illiger, 1811), con notas sobre su hábitat. Revista Forestal del Perú 6(1-2): 1-25.

Hofmann, R; Otte, K; Ponce, CF; Ríos, M. 1983. El Manejo de la Vicuña Silvestre. Eschborn, Alemania. GTZ. 705 p.

Holdridge, LR. 1947. Ecología Basada en Zonas de Vida Natural (Edición 1982). San José, Costa Rica. IICA. 216 p.

Kareiva, P; Marvier, M; Lalasz, R. 2012. Conservation in the Anthropocene. Beyond Solitude and Fragility (en línea). Breakthrough Journal. Consultado 23 nov. 2018. Disponible en https://thebreakthrough.org/index. php/journal/past-issues/issue-2/conservation-in-the-anthropocene 
Krosby, M; Tewksburky, J; Haddad, N; Hoekstra, J. 2010. Ecological Connectivity for a Changing Climate. Conservation Biology 24(6):1686-1689. DOI: https://doi. org/10.1111/j.1523-1739.2010.01585.x

Lamas, G. 1979. Algunas reflexiones y sugerencias sobre la creación de parques nacionales en el Perú. Ciencias 71(1):101-114.

Lane DF; Kratter, A; O'Neill, JP. 2017. A new species of manakin (Aves: Pipridae; Machaeropterus) from Peru with a taxonomic reassessment of the Striped Manakin (M. regulus) complex. Zootaxa 4320(2):379-390. DOI: https://doi.org/10.11646/zootaxa.4320.2.11

Laurance, W. 2013. Does research help to safeguard protected areas? Trends in Ecology and Evolution 28(5):261-266.

Laurance, WF; Camargo, JLC; Luizao, RCC; Laurance, SG; Pimm, SL; Bruna, EM; Stouffer, PC; Williamson, B; Benítez-Malvido, J; Vasconcelos, HL; Van Houtan, KS; Zartman, CE; Boyle, SA; Didham, RK; Andrade, A; Lovejoy, TE. 2011. The fate of Amazonian forest fragments: A 32-year investigation. Biological Conservation 144(1):56-67. DOI: http://dx. doi.org/10.1016/j.biocon.2010.09.021

Lehr, E; von May, R. 2017. A new species of terrestrial-breeding frog (Amphibia, Craugastoridae, Pristimantis) from high elevations of the Pui Pui Protected Forest in central Peru. Zookeys 660:17-42. DOI: http://dx.doi. org/10.3897/zookeys.660.11394

Leo, M. 1982. Conservation of the yellow-tailed woolly monkey (Lagothrix flavicauda) in Perú. Int. Zoo Yearb. 22:47-52.

Lovejoy, TE; Oren, DC. 1981. The minimum critical size of ecosystems (island biogeography theory). Forest island dynamics in man-dominated landscapes. Springer. p. 7-12.

Maass, JM; Jardel, E; Martínez-Yrízar, A; Carderón, L; Castillo, A; Euán-Ávila, J; Equihua, M. 2010. Las áreas naturales protegidas y la investigación ecológica de largo plazo en México. Ecosistemas 19(2):69-83.

Machlis, GE; Tichnell, DL. 1985. The State of the World's Parks: An international assess- ment of resources management. Westview, Boulder. $129 \mathrm{p}$.

Medina, CE; Pino, K; Pari, A; Llerena, G; Zeballos, H; López, E. 2016. Mammalian diversity in the Savanna from Peru, with three new additions from country. Papéis Avulsos de Zoologia 56(2):9-26. DOI: https://doi. org/10.11606/0031-1049.2016.56.02

Mittermeier, RA; de Macedo-Ruíz, H; Luscombe, A; Cassidy, J. 1977. Rediscovery and conservation of the Peruvian yellow-tailed woolly monkey (Lagothrix flavicauda). In Prince Rainer III of Monaco, HSH; Bourne, GH (eds.). Primate Conservation. New York, USA. Academic Press, New York. p. 95-115.

Moncrieff, AE; Johnson, O; Lane, DF; Beck, JR; Angulo, F; Fagan, J. 2017. A new species of antbird (Passeriformes: Thamnophilidae) from the Cordillera Azul, San Martín, Peru. The Auk 135(1):114-126. DOI: https://doi.org/10.1642/ $\underline{\text { AUK-17-97.1 }}$

Montambault, JR (ed.). 2002. Informes de las evaluaciones biológicas de Pampas del Heath, Perú, Alto Madidi, Bolivia, y Pando, Bolivia. Washington, USA. Conservation International. 125 p.

National Research Council. 1982. Ecological aspects of development in the humid tropics. Washington DC, USA. The National Academy Press. 297 p.

Olivera, D; Vásquez, P. 2017. Comparación entre dos métodos de estimación de edad del venado de cola blanca (Odocoileus virginianus) con fines de manejo cinegético. Revista Forestal del Perú 32(1):4-12. DOI: http://dx.doi. org/10.21704/rfp.v32i1.1032

Otte, KC. 1978. Untersuchungen zur Biologie Des Mohren Kaiman (Melanosunchus niger Spix 1825) K.C. Otte, Mun. 311 p.

Pádua, MT; Coimbra-Filho, A. 1979. Os Parques Nacionais do Brasil. Madrid, España. ICAFO. $224 \mathrm{p}$.

Piazza, A. 1967. Proyecto de establecimiento de una reserva nacional de caza, pesca y forestal y creación de una estación de investigaciones 
biológica-energética y de crianza experimental de animales silvestres en las zonas de los ríos Pacaya y Samiria. Lima, Perú. Servicio Forestal y de Caza, Documento de Trabajo (mecanografiado).

Pierret, PV. 1964. Informe I sobre la vida silvestre en el Altiplano y la Selva del Perú. Lima, Perú. Instituto de Investigaciones Forestales, Universidad Nacional Agraria, La Molina (mecanografiado).

Pierret, PV. 1965. Informe II sobre la vida silvestre en el Altiplano y la Selva del Perú. Lima, Perú. Instituto de Investigaciones Forestales, Universidad Nacional Agraria La Molina (mecanografiado).

Pitman, N; Smith, RC; Vriesendorp, C; Moskovits, D; Piana, R; Knell, G; Wachter, T (eds.). 2004. Perú: Ampiyacu, Apayacu, Yaguas, Medio Putumayo. Rapid Biological Inventories Report 12. Chicago, Illinois, USA. The Field Museum. 273 p.

Quammen, D. 1996. National parks: Nature's dead end (en línea). The New York Times. Consultado 23 nov. 2018. Disponible en https://www.nytimes.com/1996/07/28/opinion/national-parks-nature-s-dead-end.html

Rodriguez, LO; Catenazzi, A. 2017. Four new species of terrestrial-breeding frogs of the genus Phrynopus (Anura: Terrarana: Craugastoridae) from Río Abiseo National Park, Peru. Zootaxa 4273(3): 381-406. DOI: https://doi. org/10.11646/zootaxa.4273.3.4

Sanchez, PA; Buol, SW. 1975. Soils of the tropics and the world food crisis. Science 188:821827.

Sayre, R; Roca, E; Sedaghatkish, G; Young, B; Roca, R; Sheppard, S. 1999. Nature in Focus: Rapid Ecological Assessment. New York, USA. Island Press. 202 p.

Schulenberg, TS; Awbrey, K (eds.). 1997. The Cordillera del Condor of Ecuador and Peru: A Biological Assessment. RAP Working Papers 7:1-231.

Soini, P. 1997. Ecología y manejo de quelonios acuáticos en la amazonia peruana. In Fang,
T; Bodmer, R; Aquino, R; Valqui, M (eds.). Manejo de Fauna Silvestre en la Amazonia. p. 167-173. Consultado 23 nov. 2018. Disponible en http://www.comfauna.org/wp-content/uploads/2012/PDFs-Manejofaunasilvestre/Iquitos-1995/psoini EcologiaManejoQuelonidosAcuaticosAmazoniaPeruana.pdf

Tickell, O. 2013. Amazon: 441 new species discovered in four years (en línea). Ecologist, The journal for the post-industrial age. Consultado 23 nov. 2018. Disponible en http:// www.theecologist.org/News/news round up/2129973/amazon 441 new species discovered in four years.html

Tosi, JA. 1960. Zonas de Vida Natural en el Perú. Lima, Perú. Instituto Interamericano de Ciencias Agrícolas, Zona Andina (Informe N0 5). $271 \mathrm{p}$.

Tovar, A; Arnillas, CA; Eckhardt, K; Regal, F; Véliz, C. 2009. Sierra del Divisor: Una Sierra al Este del Ucayali. Lima, Perú. CONCYTEC, Centro de Datos para la Conservación. 164 p.

Vásquez, P; Justo, M. 2009. La fauna silvestre del Coto de Caza El Angolo. Guía para la identificación de las aves. Lima, Perú. Centro de Datos para la Conservación, Universidad Nacional Agraria La Molina. 201 p.

Vásquez, LC; Matallana, CL. 2016. ¿Responden las investigaciones en las áreas protegidas de Risaralda a las necesidades de manejo y gestión de la biodiversidad? Ambiente y Desarrollo 20(38):27-40.

Vilchez, MS. 1968. Parques Nacionales del Perú. Lima, Perú. Ed. La Promotora. 128 p.

WCED (World Commission on Environment and Development). 1987. Our Common Future. World Commission on Environment and Development. Oxford Univ. Press. 400 p.

WWF (World Wildlife Found) 2009. Amazon alive! A decade of discovery 1999-2009. Brasilia, DF, Brasil. World Wildlife Fund. 57 p.

Zamora, C. 1971. Capacidad de uso de los suelos del Perú (3ra aprox.). Lima, Perú. Oficina Nacional de Evaluación de Recursos naturales, ONERN. 57 p. 
Zeballos, H; Pino, K; Medina, CE; Pari, A; Chávez, D; Tinoco, N; Ceballos, G. 2018. A New Species of Small-eared Shrew of the Genus Cryptotis (Mammalia, Eulipotyphla, Soricidae) from the northernmost Peruvian Andes. Zootaxa 4377(1):51-73. DOI: https://doi. org/10.11646/zootaxa.4377.1.4

Presentado: $23 / 10 / 2018$

Aceptado: 07/12/2018

Publicado en línea: 28/12/2018 